\title{
Novel Frameshift CHD7 Mutation Related to CHARGE Syndrome
}

\author{
E. Martínez-Quintana ${ }^{a} \quad$ F. Rodríguez-González ${ }^{C} \quad$ P. Garay-Sánchez ${ }^{b}$ \\ A. Tugores ${ }^{b}$ \\ ${ }^{a}$ Cardiology Service and ${ }^{b}$ Research Unit, Insular-Materno Infantil University Hospital, and c Ophtalmology Service, \\ Dr. Negrín University Hospital of Gran Canaria, Las Palmas de Gran Canaria, Spain
}

\section{Key Words}

Abnormalities - CHARGE syndrome $\cdot$ CHD7 gene $\cdot$

Frameshift · Heart · Mutation

\begin{abstract}
CHARGE syndrome is a rare congenital condition characterized by 6 cardinal features: coloboma, heart defect, atresia choanae, retarded growth and development, genital anomalies, and ear anomalies/deafness. Mutations of the chromodomain helicase DNA-binding protein gene CHD7 are reported to be a major cause of CHARGE syndrome. Herein, we report the case of a 27-year-old patient presenting with typical symptoms who bears a novel heterozygous insertion in exon 2 of the $C H D 7$ gene (c.327dupC) resulting in an amino acid substitution and a frameshift (p.Val110Argfs*22) that leads to a 131-amino-acid truncated polypeptide, likely representing a null allele. Parental genetic screening confirmed the sporadic origin of the mutation. @ 2013 S. Karger AG, Basel
\end{abstract}

CHARGE syndrome (OMIM 214800) is a rare syndrome affecting less than 1 in 10,000 live neonates, although it is believed to be under-diagnosed due to the existence of incomplete forms [Issekutz et al., 2005]. The disease variably involves several organ systems such as the brain, eye, ear, nose, and mediastinal organs. Indeed, the name CHARGE is a mnemonic for coloboma of the eye, heart defects, atresia of the choanae, retardation of growth and development, genital or urinary abnormalities, and ear abnormalities or deafness. In choosing this acronym, the intent was to emphasize that this clustering of associated malformations occurs more frequently together than one would expect on the basis of chance.

Most cases of CHARGE syndrome are sporadic and due to de novo mutations in the gene $\mathrm{CHD}$, encoding the chromodomain helicase DNA-binding protein 7 on chromosome 8q12. Both loss of function and dominant-negative alleles of $C H D 7$ have been associated with the CHARGE phenotype [Bajpai et al., 2010; Janssen et al., 2012], so the disease may be inherited as a dominant trait [Janssen et al., 2012]. Heritability of the disease may also result from parental mosaicism, which may appear in the germline and be absent in peripheral blood cells, therefore escaping standard detection methods [Janssen et al., 2012]. There is also evidence that the CHARGE syndrome may be caused by mutations in the semaphorin-3E gene on chromosome 7q21.

Despite the current knowledge about the genetic etiology of the syndrome, there are still numerous cases where mutation detection in both genes fails to reveal a causative mutation. Furthermore, mutations in $\mathrm{CHD} 7$ are also independently associated with autosomal dominant hypogonadotropic hypogonadism 5 or Kallman syndrome (MIM 612370), adding further complexity to the clinical

\section{KARGER}

E-Mail karger@karger.com

www.karger.com/msy
(C) 2013 S. Karger AG, Basel

$1661-8769 / 13 / 0051-0036 \$ 38.00 / 0$
Efrén Martínez-Quintana

Servicio de Cardiología, Complejo Hospitalario Universitario Insular-Materno Infantil Avenida Marítima del Sur s/n ES-35016 Las Palmas de Gran Canaria (Spain)

E-Mail efrencardio@gmail.com 
Table 1. Clinical diagnosis of CHARGE syndrome according to the criteria of Blake et al. [1998] and Verloes [2005]

\begin{tabular}{|c|c|c|c|}
\hline & Major criteria & Minor criteria & Inclusion rule \\
\hline $\begin{array}{l}\text { Blake et al. } \\
\text { [1998] }\end{array}$ & $\begin{array}{l}\text { 1. Coloboma: iris, retina, choroid, or disc } \\
\text { microphthalmia } \\
\text { 2. Choanal atresia: } \\
\text { - unilateral/bilateral } \\
\text { - membranous/bony } \\
\text { - stenosis/atresia } \\
\text { 3. Ear abnormalities } \\
\text { - external ear } \\
\text { - middle ear (ossicles) } \\
\text { - inner ear (cochlea and vestibular } \\
\text { system) } \\
\text { 4. Cranial nerve dysfunction (I: anosmia, } \\
\text { VII: facial palsy, VIII: sensorineural } \\
\text { deafness and vestibular problems, IX } \\
\text { and/or X: swallowing problems) }\end{array}$ & $\begin{array}{l}\text { 1. Genital hypoplasia (micropenis, cryptorchi- } \\
\text { dism, hypoplastic labia, delayed/incomplete } \\
\text { pubertal development) } \\
\text { 2. Developmental delay (hypotonia, language } \\
\text { delay, intellectual disability) } \\
\text { 3. Cardiovascular malformations (including } \\
\text { conotruncal defects such as tetralogy of } \\
\text { Fallot, auriculoventricular canal defects and } \\
\text { aortic arch anomalies) } \\
\text { 4. Growth deficiencies (short stature) } \\
\text { 5. Orofacial cleft (cleft lip and/or palate) } \\
\text { 6. Tracheoesophageal fistula } \\
\text { 7. Characteristic face }\end{array}$ & $\begin{array}{l}4 \text { majors or } 3 \text { majors } \\
+3 \text { minors }\end{array}$ \\
\hline
\end{tabular}

consequences of $\mathrm{CHD} 7$ mutations. Thus, the diagnosis of the syndrome is still largely based on medical features, even though no single feature is universally present or sufficient for clinical diagnosis [Zentner et al., 2010].

\section{Case Report}

We report the case of a 27-year-old male patient with the diagnosis of typical CHARGE syndrome (table 1) [Verloes, 2005]. The patient underwent surgery during childhood for a bilateral cleft lip repair and presented with complete cleft palate, bilateral coronal synostosis, congenital dislocation of both hips, and persistent ductus arteriosus with a discrete subaortic fibrous ring. The patient was also receiving testosterone treatment secondary to a hypogonadotropic hypogonadism with associated bilateral cryptorchidism and testicular atrophy. No previous genetic syndromes were reported in his family background, and the patient had a normal 46,XY karyotype. Although intellectual disability was seen, the patient presented with slight growth retardation $(168 \mathrm{~cm}$ height and $57 \mathrm{~kg}$ weight).

On physical examination, the patient presented with plagiocephaly with scars secondary to previous craniotomies, broad forehead, short neck, broad nasal base with repaired cleft lip and palate, normal eyebrows, eyelashes and hair, hypertelorism with exophthalmos, and bilateral ear abnormalities (fig. 1A, B). Also, after a left ocular trauma, the patient showed a retinal detachment with an associated band keratopathy and high myopia chorioretinitis in the right eye. Dentition was normal with no dental caries. No digital abnormalities were observed.

A cerebral magnetic resonance imaging revealed moderate dilatation of the ventricular system without hydrocephalus, mild cerebral atrophy (fig. 1C), intervertebral osteochondrosis of C4-C5, C5C6 and C6-C7, C4-C5 discrete right neural foramen stenosis without radicular compression (fig. 1D), left orbital cavity hypoplasia with proptosis of the right eyeball, and hypoplasia of the turbinates.

Fibrobronchoscopy showed right septoturbinal synechiae secondary to a previous rhinoplasty with normal nasopharynx and nostrils. The patient also suffered from a total loss of smell since he was a child with no previous data of inflammation or injury.

Temporal bone computerized tomography showed bilateral mastoid air cell sclerosis, normal pneumatized tympanic cavities, hammer head sclerosis with partial anvil and total stirrup aplasia (fig. 2A), complete absence of the semicircular canals (fig. 2B), bilateral hypoplasia of the cochleas (fig. 2C), and dysplasia of both vestibules (fig. 2D). These findings are more pronounced in the left ear. Liminal pure tone audiometry performed in a free sound field, without hearing aids, indicated a profound hearing loss in both ears: the right ear revealed an air conduction between 101-110 dB and a bone conduction between $40-80 \mathrm{~dB}$, and the left ear had an air conduction loss of $120 \mathrm{~dB}$ and no bone conduction at all. A twosyllable word test in a free-field $(65 \mathrm{~dB})$, with the hearing aid in the 
right ear, showed a $0 \%$ discrimination, increasing to $50 \%$ with lip reading. No previous history of otitis was referred.

Abdominal ultrasound showed normal gallbladder, spleen, pancreas, and kidneys. The electrocardiogram showed sinus rhythm with left ventricular hypertrophy, and the transthoracic echocardiogram evidenced a subaortic discrete fibrous ring (fig. 3) with moderate subaortic gradient (echocardiographic peak gradient of $64 \mathrm{~mm}$ $\mathrm{Hg}$ and mean gradient of $37 \mathrm{~mm} \mathrm{Hg}$ ), mild-moderate aortic regurgitation, a bicuspid aortic valve, and left ventricular hypertrophy.

The patient was under testosterone treatment since he was 13 years old due to a hypogonadotropic hypogonadism with associated bilateral cryptorchidism and testicular atrophy. Serum follicle stimulating hormone levels were $0.06 \mathrm{mU} / \mathrm{ml}$ (normal range: 1.5 $14 \mathrm{mU} / \mathrm{ml}$ ), and luteinizing hormone was below $0.07 \mathrm{mU} / \mathrm{ml}$ (normal range: $1.4-7.7 \mathrm{mU} / \mathrm{ml}$ ). Luteinizing hormone-releasing hormone stimulation test showed $0.04 \mathrm{ng} / \mathrm{ml}$ of free testosterone (normal range: $0-0.9 \mathrm{ng} / \mathrm{ml})$. Replacement therapy with a combination of testosterone propionate and testosterone enanthate $(250 \mathrm{mg} \mathrm{ev}$ ery 21 days) resulted in a concentration of testosterone of $1.82 \mathrm{ng} /$ ml. Serum glucose, creatinine, cholesterol, thyroid-stimulating hormone, and cortisol levels were normal.

Exons and flanking regions of the CHD7 gene were subjected to mutation detection analysis. Heterozygote sequencing of the patient's genomic DNA isolated from peripheral blood cells revealed a cytosine duplication (GRCh37.p10; chr8; rs61654318) within the coding sequence of exon 2 at nucleotide 327 from the translation start site (c.327dupC). This results in a valine to arginine change at protein position 110 and a shift in the reading frame that caused a premature stop 21 amino acids downstream of this position (NP_060250.2; p.Val110Argfs*22). The resulting polypeptide would be devoid of any predicted functional domain of the CHD7 protein. This mutation was absent in genomic DNA isolated from peripheral blood cells from both parents and an unaffected sibling.

\section{Discussion}

CHARGE syndrome results from a dysblastogenetic and dysneurulative process mostly caused by heterozygous mutations in the $\mathrm{CHD} 7$ gene, shown to be essential for the formation of multipotent migratory neural crest cells [Bajpai et al., 2010]. Indeed, the CHARGE phenotype is supposed to result from abnormal differentiation of cephalic mesoderm and ectoderm, abnormal setting and interaction of neural crests with the cephalic mesoderm and the developing forebrain, and altered development of the rhombencephalon out of which the neural crest cells have migrated during the 3rd and 9th week of gestation [Lin et al., 1990].

Regardless of the 3 major criteria suggested by Verloes [2005], which refer to 3 nonoverlapping embryological territories (otic placode, optic vesicle and midcranial medial structures), characteristic CHARGE syndrome ear may be seen in $80-100 \%$ of the patients. While semicircular canal anomalies show a high specificity for this syn-
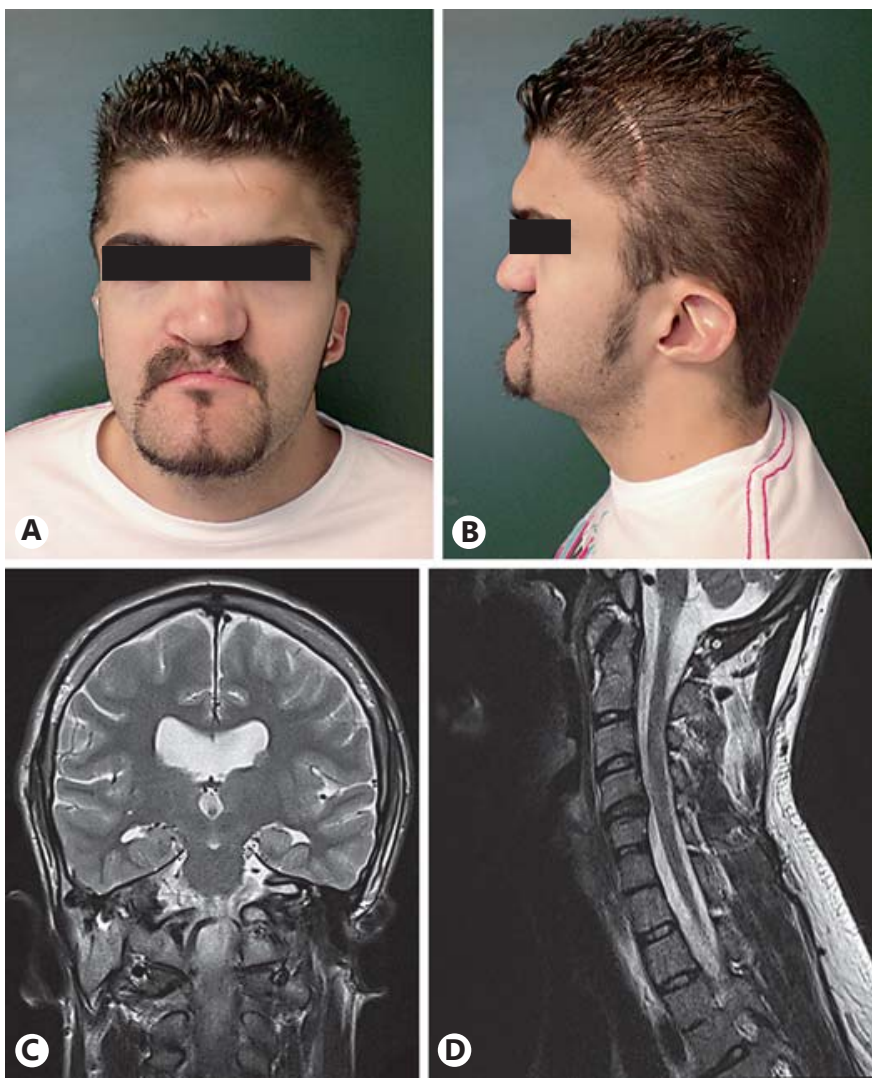

Fig. 1. Anterior (A) and lateral (B) views of the patient's face showing normal hair, broad forehead, short neck, broad nasal base with repaired cleft lip and palate, normal eyebrows and eyelashes, bilateral microtia with low-set ears, hypoplastic lobule, narrow helix, prominent antihelix, and a triangular concha. It also shows a scar secondary to a previous craniectomy. C Cerebral magnetic resonance imaging coronal view revealing moderate dilatation of the ventricular system without hydrocephalus and mild cerebral atrophy. D Cerebral magnetic resonance imaging showing intervertebral osteochondrosis of C4-C5, C5-C6 and C6-C7, and C4-C5 with discrete right neural foramen stenosis.

drome, cochlear defects do not, as the latter can also be seen in nonsyndromic deafness and in many other unrelated disorders. Concerning eye malformations, unilateral or bilateral coloboma of the iris, retina, choroid, or disc, with or without microphthalmos, are found in 80 $90 \%$ of CHARGE patients. Retinal colobomas are much more prevalent than iris colobomas and are associated with microphthalmia in those more severe cases. Nonetheless, ocular colobomas can also be seen in isolation and in an impressive number of multisystem syndromes. Finally, unilateral or bilateral choanal atresias or stenoses can be seen in $50-60 \%$ of the patients with CHARGE syndrome. However, when orofacial clefting is present, the 

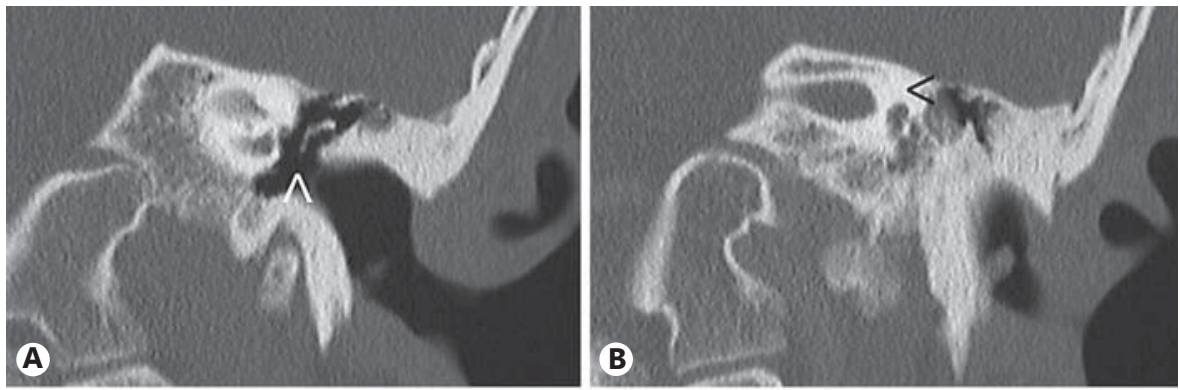

Fig. 2. Left ear computed tomography showing partial anvil and total stirrup aplasia (arrowhead in $\mathbf{A}$ ), complete absence of the semicircular canals (arrowhead in B), bilateral hypoplasia of the cochlea (arrowhead in C) with a diminishment in size of the basal and apical turns, and dysplasia of the vestibule (asterisk in D).
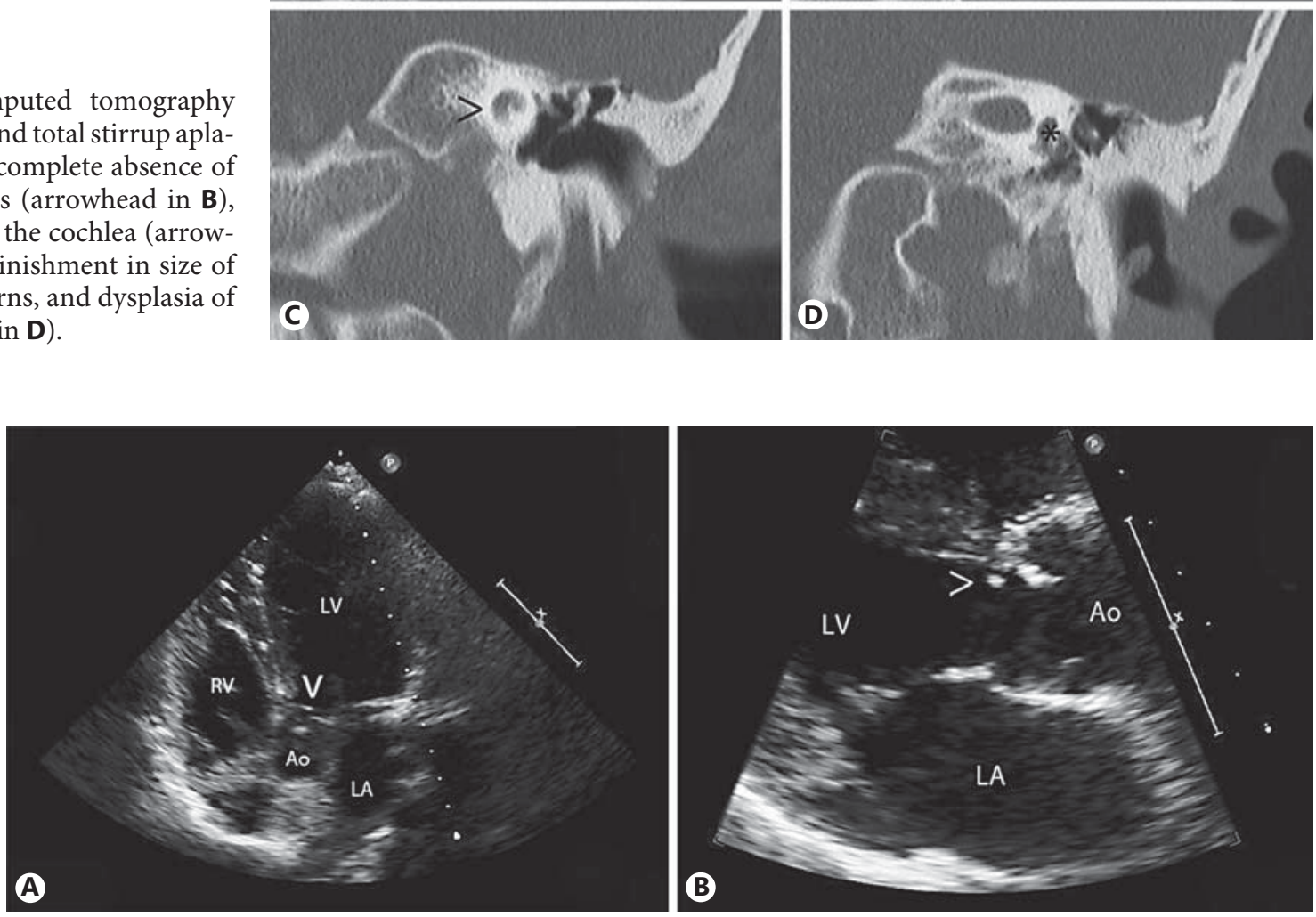

Fig. 3. Two-dimensional 4-chamber (A) and long paraesternal echocardiographic view (B) showing subaortic discrete fibrous ring (arrowheads). LV = Left ventricle; $\mathrm{RV}=$ right ventricle; $\mathrm{LA}=$ left atrium; Ao = aorta.

choanae are usually patent, so cleft lip and/or palate may be a substitute for a choanal atresia [Blake et al., 1998]. Nonetheless, stenoses of choanae may be also encountered as an isolated birth defect or as a part of other uncorrelated disorders such as chromosome abnormalities, craniosynostosis disorders or Treacher Collins syndrome [Lalani et al., 1993]. On the other hand, the 5 minor criteria suggested by Verloes [2005] (table 1) were selected as an attempt to define CHARGE by primary, not overlapping disturbances, rather than by secondary features.

CHD7 belongs to a large family of evolutionarily conserved proteins thought to play a general role in chromatin organization, therefore affecting a large number of de- velopmental pathways, which would explain the pleiotropic nature of the phenotypic spectrum of CHARGE mutations [Sanlaville and Verloes, 2007]. Over 500 mutations have been found throughout the CHD7 gene [Janssen et al., 2012]. Of these, which are predominantly heterozygous point mutations, approximately $46 \%$ are nonsense, $24 \%$ cause frameshifts, $15 \%$ are missense, $10 \%$ affect splice sites, and 3\% are intronic mutations. Only $1 \%$ are chromosomal rearrangements, and another $1 \%$ are caused by whole-gene or larger chromosomal deletions [Jongmans et al., 2006; Zentner et al., 2010; Janssen et al., 2012]. Both haploinsufficiency, caused by loss-of-function alleles, and dominant negative forms of the protein 
are believed to be responsible for the CHARGE phenotype [Bajpai et al., 2010; Bergman et al., 2011]. In the case we describe, we have found a novel insertion in exon 2 of the CHD7 gene, leading to a frameshift that results in a short polypeptide devoid of any known functional domain, and therefore, it is unlikely to be a dominant negative form of the protein. Therefore, we hypothesize that this mutation represents a null allele, causing disease due to haploinsufficiency. This is in agreement with previous findings suggesting that a full genetic dosage is required for complete function of CHD7 [Bergman et al., 2012].

This novel mutation was sporadic, as in most CHARGE cases described to date, therefore discarding family inheritance with asymptomatic parents [Pauli et al., 2009]. Heterozygous sequencing of DNA isolated from peripheral blood from both parents discarded somatic mosaicism, although germline mosaicism can not be discarded and could be the origin of the mutation as it has been described in similar cases [Janssen et al., 2012]. In this context, when a parent of the proband has CHARGE syndrome or has a CHD7 mutation, the risk to the sibs of inheriting the mutation is $50 \%$. Meanwhile, if neither parent is affected, the empiric risk is approximately $1-2 \%$, most likely attributable to germline mosaicism [Lalani et al., 1993].

The patient presented with a clear CHARGE phenotype, similarly to other patients described with other frameshift mutations in exon 2 [Lalani et al., 2006], enlarging the spectrum of mutations leading to CHARGE syndrome. Heterozygous mutations in the $\mathrm{CHD} 7$ gene may be found, depending on the patient cohort analyzed, in over $95 \%$ of the patients [Jongmans et. al, 2008] who fulfill the criteria of Blake et al. [1998] or Verloes [2005] (table 1). CHD7 mutation-positive individuals present more frequently with inner ear malformations, facial nerve palsy and ocular colobomas, while mutation-negative patients are less likely to present with delayed growth and development alterations. However, no significant differences have been seen between mutation-positive and mutation-negative patients in relation to heart defects, choanal atresia and endocrine, urogenital, spinal, and skeletal abnormalities [Zentner et al., 2010].

Due to the clinical variability of the CHARGE syndrome and because most of the patients with a $\mathrm{CHD} 7 \mathrm{mu}-$ tation fulfill the criteria of Blake et al. [1998] or Verloes [2005] (table 1), mutation screening of the CHD7 gene should be emphasized as a useful tool in patients with clinical suspicion. This is especially important in patients with mild CHARGE phenotype, asymptomatic or mosaic parents which may lead to parental transmission or patients diagnosed with Kallmann syndrome or hypogonadotropic hypogonadism presenting with minimal additional features of CHARGE syndrome [Blake et al., 2011].

\section{References}

Bajpai R, Chen DA, Rada-Iglesias A, Zhang J, Xiong Y, et al: CHD7 cooperates with PBAF to control multipotent neural crest formation. Nature 463:958-962 (2010).

-Bergman JE, Janssen N, Hoefsloot LH, Jongmans MC, Hofstra RM, van Ravenswaaij-Arts CM: CHD7 mutations and CHARGE syndrome: the clinical implications of an expanding phenotype. J Med Genet 48:334-342 (2011).

- Bergman JE, Janssen N, van der Sloot AM, de Walle HE, Schoots J, et al: A novel classification system to predict the pathogenic effects of $\mathrm{CHD} 7$ missense variants in CHARGE syndrome. Hum Mutat 33:1251-1260 (2012).

Blake K, van Ravenswaaij-Arts CM, Hoefsloot L, Verloes A: Clinical utility gene card for: CHARGE syndrome. Eur J Hum Genet 19 (2011).

Blake KD, Davenport SLH, Hall BD, Hefner MA, Pagon RA, et al: CHARGE association: an update and review for the primary pediatrician. Clin Pediatr 37:159-173 (1998).
Issekutz KA, Graham JM Jr, Prasad C, Smith IM, Blake KD: An epidemiological analysis of CHARGE syndrome: preliminary results from a Canadian study. Am J Med Genet 133A:309-317 (2005).

Janssen N, Bergman JE, Swertz MA, Tranebjaerg L, Lodahl M, et al: Mutation update on the CHD7 gene involved in CHARGE syndrome. Hum Mutat 33:1149-1160 (2012).

Jongmans MC, Admiraal RJ, van der Donk KP, Vissers LE, Baas AF, et al: CHARGE syndrome: the phenotypic spectrum of mutations in the CHD7 gene. J Med Genet 43:306314 (2006)

Jongmans MC, Hoefsloot LH, van der Donk KP, Admiraal RJ, Magee A, et al: Familial CHARGE syndrome and the CHD7 gene: a recurrent missense mutation, intrafamilial recurrence and variability. Am J Med Genet A 146A:43-50 (2008).

-Lalani SR, Hefner MA, Belmont JW, Davenport SLH: CHARGE Syndrome, in Pagon RA, Adam MP, Bird TD, Dolan CR, Fong C-T, Stephens K (eds): GeneReviews (University of Washington, Seattle 1993). http://www.ncbi. nlm.nih.gov/pubmed/20301296.
Lalani SR, Safiullah AM, Fernbach SD, Harutyunyan KG, Thaller C, et al: Spectrum of CHD7 mutations in 110 individuals with CHARGE syndrome and genotype-phenotype correlation. Am J Hum Genet 78:303-314 (2006).

Lin AE, Siebert JR, Graham JM Jr: Central nervous system malformations in the CHARGE association. Am J Med Genet 37:304-310 (1990).

Pauli S, Pieper L, Häberle J, Grzmil P, Burfeind P, et al: Proven germline mosaicism in a father of two children with CHARGE syndrome. Clin Genet 75:473-479 (2009).

- Sanlaville D, Verloes A: CHARGE syndrome: an update. Eur J Hum Genet 15:389-399 (2007).

Verloes A: Updated diagnostic criteria for CHARGE syndrome: a proposal. Am J Med Genet 133A:306-308 (2005).

Zentner GE, Layman WS, Martin DM, Scacheri PC: Molecular and phenotypic aspects of CHD7 mutation in CHARGE syndrome. Am J Med Genet A 152A:674-686 (2010).
40
Mol Syndromol 2014;5:36-40 DOI: $10.1159 / 000355431$
Martínez-Quintana/Rodríguez-González/ Garay-Sánchez/Tugores 